'Sección Cardiología, Servicio de Medicina Interna. Hospital del Salvador. Santiago, Chile. ${ }^{2}$ Departamento de Medicina Interna Oriente. Escuela de Medicina, Universidad de Chile. Santiago, Chile. ${ }^{3}$ Facultad de Medicina, Universidad de los Andes. Santiago, Chile. aBecario programa formación Cardiología.

bBecario Programa de formación de especialista en Cardiología. 'Enfermera.

Trabajo no recibió financiamiento. Los autores declaran no tener conflictos de interés.

Recibido el 8 de junio de 2018, aceptado el 8 de enero de 2019.

Correspondencia a: Víctor Rossel vrossel@med.uchile.cl

\section{Pacientes elegibles para las nuevas terapias de la insuficiencia cardíaca en un policlínico especializado}

\author{
VÍCTOR ROSSEL ${ }^{1,2}$, MANUEL DUARTE $^{2, \mathrm{a}}$, PILAR MUÑOZ ${ }^{1,2}$, \\ CATHERINE BRAVO ${ }^{1}$, GUSTAVO BOBADILLA ${ }^{3, b}$, \\ FERNANDO VERDUGO ${ }^{2, a}$, CARMEN GUARDAMAGNA ${ }^{1, c}$
}

\section{Proportion of patients with heart failure in a specialized clinic eligible for novel therapies}

Background: Pharmacological treatment improves survival in patients with heart failure with reduced ejection fraction. The use of sacubutril/valsartan and ivabradine has been recently approved and incorporated in the latest guidelines. Aim: To identify candidates eligible for these therapies among patients treated in a heart failure clinic, considering the inclusion criteria for the PARADIGM-HF and SHIFT trials. Material and Methods: Cross-sectional study on 158 patients aged $62 \pm 11$ years (67\% male) with heart failure and reduced ejection fraction, with at least three months of follow-up and without decompensation. The percentage of patients complying for the inclusion criteria for the PARADIGM-HF y SHIFT trials was determined. Results: In 37\%, the etiology of heart failure was ischemic, 49\% were in functional class I, their ejection fraction was $33 \pm$ $11 \%$ and their median Pro-brain natriuretic peptide was $800 \mathrm{pg} / \mathrm{mL}$. Ninety five percent were treated with vasodilators, $97 \%$ with beta-blockers and $82 \%$ with aldosterone antagonists. Using PARADIGM-HF and SHIFT criteria, 11 patients (7\%) were eligible for sacubitril / valsartan and 21 patients (13.3\%) for ivabradine. Among the main causes of non-eligibility for sacubitril / valsartan were being functional class I (48.7\%) and not achieving a stable dose of enalapril $\geq 20 \mathrm{mg} /$ day or losartan $\geq 100 \mathrm{mg} /$ day (24.7\%). In the case of ivabradine, apart from those in functional class I, the absence of sinus rhythm and a heart rate $<70 /$ min when receiving a maximal tolerated dose of beta-blockers, were present in 22\%. Conclusions: A low percentage of our patients were eligible for these therapies. Among the causes that explain these results were clinical stability, a high percentage of patients in functional class I and being in a disease modifying treatment.

(Rev Med Chile 2019; 147: 330-333)

Key words: Drug Therapy; Heart Diseases; Heart Failure; Ivabradine.
I a insuficiencia cardíaca (IC) es considerada actualmente un problema de salud pública de gran relevancia, tanto por la prevalencia de la enfermedad, que se estima en $3 \%$ de la población, como por su elevada morbilidad y mortalidad, la que puede alcanzar cifras de 50\% a 5 años ${ }^{1}$. Existe desde hace años un tratamiento establecido para pacientes con IC y fracción de eyección ventricular izquierda (FEVI) reducida de bloqueo neurohormonal que consiste en inhibidores de la enzima de conversión (IECA) o antagonistas del receptor de la angiotensina (ARA), beta-bloqueadores (BB) y antagonistas de aldosterona (AA). A pesar de ello, no todos 
los pacientes responden de manera satisfactoria a estas terapias, por lo que han continuado desarrollándose nuevos fármacos para mejorar aun más el pronóstico de estos pacientes, destacándose entre ellos sacubitril/valsartán e ivabradina. Sacubril/ valsatran, que posee un efecto dual, inhibidor de neprilisina y $\mathrm{ARA}^{2}$, demostró reducir hospitalizaciones por empeoramiento de la IC, mortalidad cardiovascular y mortalidad total al ser comparado con enalapril (PARADIGM-HF) ${ }^{3}$. Ivabradina reduce la frecuencia cardiaca al inhibir el canal If del nodo sinusal ${ }^{4}$, logró reducir el punto de observación combinado de mortalidad y hospitalización por IC con FEVI reducida en pacientes sintomáticos con frecuencia cardiaca (FC) $\geq 70$ / min a pesar de dosis máximas de BB $(\text { SHIFT })^{5}$. En relación con esta evidencia, ambos fármacos han sido incorporados en las últimas guías de manejo de la IC, publicadas por las sociedades europea ${ }^{6}$ y americana $^{7}$ de Cardiología. Estos fármacos, a pesar de estar disponibles en nuestro país aún no son de uso habitual. El objetivo de este trabajo es identificar entre los pacientes atendidos en un policlínico especializado de IC candidatos elegibles para estas nuevas terapias, considerando los criterios de ingreso de los trabajos mencionados en los que se demostró su efectividad y seguridad.

\section{Materiales y Método}

Se realizó un estudio de corte transversal en pacientes con IC con FEVI reducida en control en el policlínico de IC del Hospital del Salvador. La población en control corresponde a pacientes con IC con FEVI menor a $40 \%$ al momento de ingreso, en etapa $\mathrm{C}$ de la clasificación por estadios de la IC.

Se registraron variables biodemográficas, clínicas y de laboratorio de aquellos pacientes que acudieron a control al policlínico entre junio y diciembre del año 2017, que hubiesen completado al menos 3 meses de seguimiento y sin episodios intercurrentes de descompensación. Se obtuvo autorización del Comité de Ética del Servicio de Salud Metropolitano Oriente para realizar este estudio. Se estimó en base a los criterios de inclusión de los ensayos clínicos PARADIGM-HF ${ }^{3}$ y SHIFT ${ }^{5}$ el porcentaje de pacientes potenciales candidatos a recibir los medicamentos sacubitril/valsartan o ivabradina. Los criterios de inclusión considerados para recibir sacubitril/valsartan, según estudio PARADIGM-HF fueron: IC sintomática (clase funcional [CF] II-III-IV), FEVI $\leq 40 \%$ con niveles de propéptido natriurético cerebral (Pro-BNP) $\geq 600 \mathrm{pg} / \mathrm{ml}$ o, en caso de hospitalización por IC en los últimos 12 meses $\geq 400 \mathrm{pg} / \mathrm{ml}$, una velocidad de filtración glomerular estimada (VFGe) $\geq 30 \mathrm{ml} /$ $\mathrm{min} / 1,73 \mathrm{~m}^{2}$ y que pudieran tolerar tratamiento con enalapril ( $\geq 20 \mathrm{mg} /$ día) o su equivalente para losartán ( $\geq 100 \mathrm{mg} /$ día). Los criterios de inclusión para recibir ivabradina se basaron en los criterios de ingreso al ensayo clinico SHIFT: IC sintomática (CF II-III-IV), FEVI $\leq 35 \%$, ritmo sinusal y una $\mathrm{FC} \geq 70 /$ min con uso de $\mathrm{BB}$ en la dosis máxima tolerada. Las variables continuas se expresan en promedios \pm desviación estándar y las categóricas en porcentajes. Se calcularon los porcentajes para cada uno de los criterios mencionados en los ensayos clínicos y, luego, el porcentaje final que resultó al combinar en forma sucesiva los diferentes criterios considerados para recibir sacubitril/ valsartán o ivabradina en los estudios respectivos.

\section{Resultados}

Se incluyeron en este estudio 158 pacientes (Tabla 1); edad $62 \pm 11$ años; 67\% género masculino; $36,7 \%$ etiología isquémica; CF I 48,7\%; FEVI $33 \pm 11 \% ; 94,8 \%$ en tratamiento con vasodilatadores ( $51,8 \%$ enalapril, dosis promedio 20 $\pm 13 \mathrm{mg} /$ día y $39,2 \%$ losartán, dosis promedio 69 $\pm 32 \mathrm{mg} /$ día; otros vasodilatadores 3,8\%); 97,4\% con $\mathrm{BB},(69,6 \%$ con carvedilol, dosis promedio $30 \pm 16 \mathrm{mg}$ y $27,8 \%$ con bisoprolol, dosis promedio $6 \pm 3 \mathrm{mg}$ ); $81,6 \% \mathrm{AA}$, (dosis promedio de espironolactona $32 \pm 12 \mathrm{mg}$ ) y diuréticos $68,3 \%$ (dosis promedio furosemida $40 \pm 22 \mathrm{mg}$ ). Al aplicar los criterios de ingreso PARADIGM-HF, 11 pacientes del policlínico (7\%) resultaron ser elegibles para iniciar sacubitril/valsartan. En el caso de ivabradina, 21 pacientes $(13,3 \%)$ cumplieron los criterios SHIFT y se consideraron elegibles para iniciar el fármaco. Entre las principales razones para ser considerados no elegibles a estas nuevas terapias, para sacubitril/valsartán encontramos: CF I $(48,1 \%)$ y no lograr una dosis de enalapril $\geq 20 \mathrm{mg} /$ día o losartán $\geq 100 \mathrm{mg} /$ día $(24,7 \%)$ y para uso de ivabradina: CF I $(48,7 \%)$ y ausencia de $\mathrm{RS}$ o $\mathrm{FC}<70 / \mathrm{min}$ en dosis máxima tolerada de BB $(22,1 \%)$ (Tabla 2$)$. 
Tabla 1. Características generales de pacientes

\begin{tabular}{|lc|}
\hline Variable & $\begin{array}{c}\text { Pacientes } \\
\text { (n = 158) }\end{array}$ \\
\hline Edad (años) & $62 \pm 11$ \\
Género masculino (\%) & 67 \\
\hline Etiología isquémica (\%) & 36,7 \\
\hline Clase funcional I (\%) & 48,7 \\
\hline FEVI (\%) & $32 \pm 11$ \\
\hline Uso de vasodilatadores (\%) & 94,8 \\
\hline Uso de betabloqueantes (\%) & 97,4 \\
\hline Uso de antagonistas de aldosterona (\%) & 81,6 \\
\hline Uso de diuréticos (\%) & 68,3 \\
\hline Uso de TCR/DAl/TRC-DAl (\%) & 37,3 \\
\hline Presión arterial sistólica (mmHg) & $120 \pm 20$ \\
\hline Frecuencia cardíaca (latidos/min) & $72 \pm 12$ \\
\hline Pro-BNP* (pg/mL) & 800 \\
\hline
\end{tabular}

FEVI: fracción de eyección de ventrículo izquierdo; TRC: terapia de resincronización cardíaca; DAl: desfibrilador automático implantable; $\mathrm{mmHg}$ : milímetros de mercurio; Pro-BNP: propétido natriurético cerebral; pg/mL: picogramos/ mililitro. *Mediana.

\section{Discusión}

En este trabajo solo 7\% de los pacientes atendidos en nuestro policlínico de IC resultaron elegibles para iniciar sacubutril/valsartan, según los criterios de inclusión de PARADIGM-HF y 13,3\% para iniciar ivabradina, según los criterios de ingreso a SHIFT. Al comparar las cifras obtenidas con lo comunicado en la literatura el porcentaje resultó menor. Pellicori et al. ${ }^{8}$ mostraron $21 \%$ de elegibilidad para sacubutril/valsartan en un policlínico de IC en Inglaterra, mientras que Norberg et $\mathrm{al}^{9}$ incorporaron a $24 \%$, en una población de IC con FEVI reducida en Suecia. El registro sueco de Das et al, publicado el $2017^{10}$, mostró una elegibilidad para ivabradina de 14,2\%. En relación con estos resultados, es necesario señalar que esta es una población seleccionada de pacientes atendida en una clínica especializada de la enfermedad, con un enfoque multiprofesional, con atención dirigida y periódica según los requerimientos clínicos, lo que contribuye a que un alto porcentaje reciba tratamiento modificador de la enfermedad, incluso con cifras superiores a las publicadas en los últimos ensayos de $\mathrm{IC}^{3}$. De los pacientes incluidos en este estudio, $95 \%$ se encontraba con terapia

Tabla 2. Criterios de no elegibilidad para nuevas terapias

\begin{tabular}{|c|c|c|}
\hline \multirow[t]{2}{*}{ Variable } & \multicolumn{2}{|c|}{ Pacientes policlínico IC $(n=158)$} \\
\hline & $\mathbf{n}$ & $\%$ \\
\hline \multicolumn{3}{|l|}{ Ensayo PARADIGM-HF (sacubitril/valsartán) } \\
\hline Clase funcional I & 76 & 48,1 \\
\hline $\mathrm{FEVI}>40 \%$ & 15 & 9,5 \\
\hline ProBNP $<600$ pg/mL, sin hospitalización o ProBNP $<400$ pg/mL con hospitalización & 15 & 9,5 \\
\hline Dosis de ENLP $<20$ mg/día o LST $<100$ mg/día & 39 & 24,7 \\
\hline Potasio $\geq 5,4 \mathrm{mEq} / \mathrm{L}$ & 2 & 1,3 \\
\hline Suma de todos los criterios de no elegibilidad & 147 & 93,1 \\
\hline \multicolumn{3}{|l|}{ Ensayo SHIFT (ivabradina) } \\
\hline Clase funcional I & 77 & 48,7 \\
\hline $\mathrm{FEVI}>35 \%$ & 25 & 13,3 \\
\hline Ausencia de $\mathrm{RS}, \mathrm{FC} \leq 70 / \mathrm{min}$, en dosis máxima de $\mathrm{BB}$ & 35 & 22,1 \\
\hline Suma de todos los criterios de no elegibilidad & 137 & 86,7 \\
\hline
\end{tabular}

ENLP: enalapril; LST: losartán; mg: miligramos; Pro-BNP: propeptido natriurético cerebral; pg/ml: picogramos por mililitro; FEVI: fracción de eyección de ventrículo izquierdo; PAS: presión arterial sistólica; VFG: velocidad de filtración glomerular; $\mathrm{mEq} / \mathrm{L}$ : miliequivalentes por litro. RS: ritmo sinusal; FC: frecuencia cardíaca; BB: betabloqueador. 
vasodilatadora, con dosis promedio de enalapril muy cercanas a las óptimas y por sobre las dosis obtenidas en los ensayos clínicos; $98 \%$ con BB, aunque con dosis promedio de carvedilol inferiores a las consideradas óptimas y a las obtenidas en los ensayos clínicos y $81 \%$ con AA, con dosis promedio de espironolactona superiores a las obtenidas en los ensayos clínicos. En relación con lo anterior, la cohorte de pacientes controlados en este policlínico muestra estabilidad clínica, con bajos niveles de Pro-BNP y prácticamente la mitad de los pacientes en CF I, lo que inmediatamente los excluyó de estas nuevas terapias.

Esta comunicación es la primera publicación en nuestro país que se refiere la proporción de pacientes elegibles para estas nuevas terapias incorporadas al tratamiento farmacológico y disponibles en Chile. Contribuye con información derivada desde la práctica clínica diaria en un policlínico de atención especializada de IC a tener una aproximación a la población de pacientes con IC y FEVI reducida susceptible de ser beneficiaria de estos tratamientos de segunda línea, recomendados en las últimas guías clínicas de la enferme$\operatorname{dad}^{6,7}$, que han demostrado beneficios en términos de morbilidad y mortalidad en reemplazo de las terapias más habituales de la enfermedad.

\section{Referencias}

1. Ho KK, Pinsky JL, Kannel WB, Levy D. The epidemiology of heart failure: the Framingham Study. J Am Coll Cardiol 1993; 22 (Octubre): 6A-13A.

2. Rademaker MT, Charles CJ, Espiner EA, Nicholls MG, Richards AM, Kosoglou T. Combined neutral endopeptidase and angiotensin-converting enzyme inhibition in heart failure: rol of natriuretic peptides and angiotensin
II. J Cardiovasc Pharmacol 1998; 31: 116-25.

3. McMurray JJ, Packer M, Desai AS, Gong J, Lefkowitz MP, Rizkala AR, et al. Angiotensin-neprilysin inhibition versus enalapril in heart failure. N Engl J Med 2014; 371: 993-1004.

4. Di Francesco D. Funny channels in the control of cardiac rhythm and mode of action of selective blockers. Pharmacol Res. 2006; 53: 399-406.

5. Swedberg K, Komajda M, Böhm M, Borer J, Ford I, Dubost-Brama A, et al. Ivabradine and outcomes in chronic heart failure (SHIFT): a randomised placebo-controlled study. Lancet 2010; 376: 875-85.

6. Ponikowski P, Voors AA, Anker SD, Bueno H, Cleland JGF, Coats AJS, et al. 2016 ESC Guidelines for the Diagnosis and Treatment of Acute and Chronic Heart Failure. Eur Heart J 2016; 37: 2129-200.

7. Yancy CW, Jessup M, Bozkurt B, , Butler J, Casey DE Jr, Colvin MM, et al. 2016 ACC/AHA/HFSA Focused Update on New Pharmacological Therapy for Heart Failure: An Update of the 2013 ACCF/AHA Guideline for the Management of Heart Failure: A Report of the American College of Cardiology/American Heart Association Task Force on Clinical Practice Guidelines and the Heart Failure Society of America. Circulation 2016; 134: e282-93.

8. Pellicori P, Urbinati A, Shah P, MacNamara A, Kazmi S, Dierckx R, et al. What proportion of patients with chronic heart failure are eligible for sacubitril-valsartan? Eur J Heart Fail 2017; 19: 768-78.

9. Norberg H, Bergdahl E, Lindmark K. Eligibility of sacubitril-valsartan in a real-world heart failure population: a community-based single-centre study. ESC Heart Fail 2018; 5: 337-43.

10. Das D, Savarese G, Dahlström U, Fu M, Howlett J, Ezekowitz J, et al. Ivabradine in Heart Failure: The Representativeness of SHIFT in a Broad Population of Patients With Chronic Heart Failure. Circ Heart Fail 2017; 10: 1-14. 